\title{
Approximate solution of intuitionistic fuzzy differential equations by using Picard's method
}

\author{
R. Ettoussi, S. Melliani and L. S. Chadli \\ Department of Mathematics \\ Laboratory of Applied Mathematics and Scientific Computing \\ Sultan Moulay Slimane University \\ PO Box 523, 23000 Beni Mellal, Morocco \\ e-mails: razika.imi@gmail.com, saidmelliani@gmail.com, \\ sa.chadli@yahoo.fr
}

Received: 1 April 2018

Accepted: 20 April 2018

\begin{abstract}
Our main result in this paper is to find the power series solution of an intuitionistic fuzzy differential equation $x^{\prime}(t)=f(t, x(t)), x\left(t_{0}\right)=x_{0}$ by using successive approximation method and we prove that the approximate solution converge uniformly in $t$ to the exact solution. Finally, we illustrate this result with a numerical example.
\end{abstract}

Keywords: Intuitionistic fuzzy solution, Intuitionistic fuzzy number, Picard's iterative method. 2010 Mathematics Subject Classification: 34A07, 03E72.

\section{Introduction}

The concept of intuitionistic fuzzy is introduced by K. Atanassov in 1983 [1,2]. This concept is a generalization of fuzzy theory introduced by L. Zadeh [8]. Intutionistic fuzzy differential equation is very rare. Melliani and Chadli [6] solve partial differential equation with intutionistic fuzzy number.

By the metric space defined in [5] we have something that makes sense to study this problem in intuitionistic fuzzy theory.

In [4] the authors discussed the existence and uniqueness of solution of the intuitionistic fuzzy differential equation, using the method of successive approximation. 
In this paper we considered Picard's approximation methods for finding approximate solution of an intuitionistic fuzzy differential equation

$$
\left\{\begin{array}{l}
x^{\prime}(t)=f(t, x(t)) \\
x\left(t_{0}\right)=x_{0}
\end{array}\right.
$$

where $x_{0}$ is an intuitionistic fuzzy quantity and $f: I \times \mathrm{IF}_{1} \rightarrow \mathrm{IF}_{1}$ is levelwise continuous.

At first, in Section 2, we give some definitions and properties regarding the concept of an intuitionistic fuzzy sets. The main results of this work is discussed in Section 3. Finally, we illustrated our theorem numerically by considering an example.

\section{Preliminaries}

Let us $T=[c, d] \subset \mathbb{R}$ be a compact interval. we denote by

$$
\mathrm{IF}_{1}=\mathrm{IF}(\mathbb{R})=\left\{\langle u, v\rangle: \mathbb{R} \rightarrow[0,1]^{2}, \mid \forall x \in \mathbb{R} 0 \leq u(x)+v(x) \leq 1\right\}
$$

An element $\langle u, v\rangle$ of $\mathrm{IF}_{1}$ is said an intuitionistic fuzzy number if it satisfies the following conditions

(i) $\langle u, v\rangle$ is normal i.e there exists $x_{0}, x_{1} \in \mathbb{R}$ such that $u\left(x_{0}\right)=1$ and $v\left(x_{1}\right)=1$.

(ii) $u$ is fuzzy convex and $v$ is fuzzy concave.

(iii) $u$ is upper semi-continuous and $v$ is lower semi-continuous

(iv) $\operatorname{supp}\langle u, v\rangle=\operatorname{cl}\{x \in \mathbb{R}: \mid v(x)<1\}$ is bounded.

so we denote the collection of all intuitionistic fuzzy number by $\mathrm{IF}_{1}$

Definition 2.1 ([5]). An intuitionistic fuzzy number $\langle u, v\rangle$ in parametric form is a pair

$$
\langle u, v\rangle=\left(\left(\underline{\langle u, v\rangle^{+}}, \overline{\langle u, v\rangle^{+}}\right),\left(\underline{\langle u, v\rangle^{-}}, \overline{\langle u, v\rangle}^{-}\right)\right)
$$

of functions $\underline{\langle u, v\rangle^{-}}(\alpha), \overline{\langle u, v\rangle}^{-}(\alpha), \underline{\langle u, v\rangle}^{+}(\alpha)$ and $\overline{\langle u, v\rangle}^{+}(\alpha)$, which satisfies the following requirements:

1. $\langle u, v\rangle^{+}(\alpha)$ is a bounded monotonic increasing continuous function,

2. $\overline{\langle u, v\rangle}^{+}(\alpha)$ is a bounded monotonic decreasing continuous function,

3. $\langle u, v\rangle^{-}(\alpha)$ is a bounded monotonic increasing continuous function,

4. $\overline{\langle u, v\rangle}^{-}(\alpha)$ is a bounded monotonic decreasing continuous function,

5. $\underline{\langle u, v\rangle}^{-}(\alpha) \leq \overline{\langle u, v\rangle}^{-}(\alpha)$ and $\underline{\langle u, v\rangle^{+}}(\alpha) \leq \overline{\langle u, v\rangle}^{+}(\alpha)$, for all $0 \leq \alpha \leq 1$. 
Example. A Triangular Intuitionistic Fuzzy Number (TIFN) $\langle u, v\rangle$ is an intuitionistic fuzzy set in $\mathbb{R}$ with the following membership function $u$ and non-membership function $v$ :

$$
\begin{aligned}
& u(x)= \begin{cases}\frac{x-a_{1}}{a_{2}-a_{1}} & \text { if } a_{1} \leq x \leq a_{2} \\
\frac{a_{3}-x}{a_{3}-a_{2}} & \text { if } a_{2} \leq x \leq a_{3}, \\
0 & \text { otherwise }\end{cases} \\
& v(x)= \begin{cases}\frac{a_{2}-x}{a_{2}-a_{1}^{\prime}} & \text { if } a_{1}^{\prime} \leq x \leq a_{2} \\
\frac{x-a_{2}}{a_{3}^{\prime}-a_{2}} & \text { if } a_{2} \leq x \leq a_{3}^{\prime}, \\
1 & \text { otherwise. }\end{cases}
\end{aligned}
$$

where $a_{1}^{\prime} \leq a_{1} \leq a_{2} \leq a_{3} \leq a_{3}^{\prime}$

This TIFN is denoted by $\langle u, v\rangle=\left\langle a_{1}, a_{2}, a_{3} ; a_{1}^{\prime}, a_{2}, a_{3}^{\prime}\right\rangle$.

Its parametric form is

$$
\begin{aligned}
& {\underline{\langle u, v\rangle^{+}}}^{+}(\alpha)=a_{1}+\alpha\left(a_{2}-a_{1}\right), \overline{\langle u, v\rangle}^{+}(\alpha)=a_{3}-\alpha\left(a_{3}-a_{2}\right) \\
& \underline{\langle u, v\rangle^{-}}(\alpha)=a_{1}^{\prime}+\alpha\left(a_{2}-a_{1}^{\prime}\right), \overline{\langle u, v\rangle}^{-}(\alpha)=a_{3}^{\prime}-\alpha\left(a_{3}^{\prime}-a_{2}\right)
\end{aligned}
$$

For $\alpha \in[0,1]$ and $\langle u, v\rangle \in \mathrm{IF}_{1}$, the upper and lower $\alpha$-cuts of $\langle u, v\rangle$ are defined by

$$
[\langle u, v\rangle]^{\alpha}=\{x \in \mathbb{R}: v(x) \leq 1-\alpha\}
$$

and

$$
[\langle u, v\rangle]_{\alpha}=\{x \in \mathbb{R}: u(x) \geq \alpha\}
$$

Remark 2.1. If $\langle u, v\rangle \in I F_{1}$, so we can see $[\langle u, v\rangle]_{\alpha}$ as $[u]^{\alpha}$ and $[\langle u, v\rangle]^{\alpha}$ as $[1-v]^{\alpha}$ in the fuzzy case.

We define $0_{\langle 1,0\rangle} \in \mathrm{IF}_{1}$ as

$$
0_{\langle 1,0\rangle}(t)= \begin{cases}\langle 1,0\rangle & t=0 \\ \langle 0,1\rangle & t \neq 0\end{cases}
$$

For $\langle u, v\rangle,\langle z, w\rangle \in \mathrm{IF}_{1}$ and $\lambda \in \mathbb{R}$, the addition and scaler-multiplication are defined as follows

$$
\begin{aligned}
& {[\langle u, v\rangle \oplus\langle z, w\rangle]^{\alpha}=[\langle u, v\rangle]^{\alpha}+[\langle z, w\rangle]^{\alpha}, \quad[\lambda\langle z, w\rangle]^{\alpha}=\lambda[\langle z, w\rangle]^{\alpha}} \\
& {[\langle u, v\rangle \oplus\langle z, w\rangle]_{\alpha}=[\langle u, v\rangle]_{\alpha}+[\langle z, w\rangle]_{\alpha}, \quad[\lambda\langle z, w\rangle]_{\alpha}=\lambda[\langle z, w\rangle]_{\alpha}}
\end{aligned}
$$

Definition 2.2. Let $\langle u, v\rangle$ an element of $I F_{1}$ and $\alpha \in[0,1]$, we define the following sets:

$$
\begin{array}{r}
{[\langle u, v\rangle]_{l}^{+}(\alpha)=\inf \{x \in \mathbb{R} \mid u(x) \geq \alpha\}, \quad[\langle u, v\rangle]_{r}^{+}(\alpha)=\sup \{x \in \mathbb{R} \mid u(x) \geq \alpha\}} \\
{[\langle u, v\rangle]_{l}^{-}(\alpha)=\inf \{x \in \mathbb{R} \mid v(x) \leq 1-\alpha\}, \quad[\langle u, v\rangle]_{r}^{-}(\alpha)=\sup \{x \in \mathbb{R} \mid v(x) \leq 1-\alpha\}}
\end{array}
$$




\section{Remark 2.2.}

$$
\begin{aligned}
& {[\langle u, v\rangle]_{\alpha}=\left[[\langle u, v\rangle]_{l}^{+}(\alpha),[\langle u, v\rangle]_{r}^{+}(\alpha)\right]} \\
& {[\langle u, v\rangle]^{\alpha}=\left[[\langle u, v\rangle]_{l}^{-}(\alpha),[\langle u, v\rangle]_{r}^{-}(\alpha)\right]}
\end{aligned}
$$

On the space $\mathrm{IF}_{1}$ we will consider the following metric,

$$
\begin{aligned}
d_{\infty}(\langle u, v\rangle,\langle z, w\rangle) & =\frac{1}{4} \sup _{0<\alpha \leq 1}\left|[\langle u, v\rangle]_{r}^{+}(\alpha)-[\langle z, w\rangle]_{r}^{+}(\alpha)\right| \\
& +\frac{1}{4} \sup _{0<\alpha \leq 1}\left|[\langle u, v\rangle]_{l}^{+}(\alpha)-[\langle z, w\rangle]_{l}^{+}(\alpha)\right| \\
& +\frac{1}{4} \sup _{0<\alpha \leq 1}\left|[\langle u, v\rangle]_{r}^{-}(\alpha)-[\langle z, w\rangle]_{r}^{-}(\alpha)\right| \\
& +\frac{1}{4} \sup _{0<\alpha \leq 1}\left|[\langle u, v\rangle]_{l}^{-}(\alpha)-[\langle z, w\rangle]_{l}^{-}(\alpha)\right|
\end{aligned}
$$

Theorem 2.1 ([7]). The metric space $\left(I F_{1}, d_{\infty}\right)$ is complete.

Definition 2.3. A mapping $F: T \rightarrow I F_{1}$ is called levelwise continuous at $t_{0} \in T$ if the set-valued mappings $F_{\alpha}(t)=[F(t)]_{\alpha}$ and $F^{\alpha}(t)=[F(t)]^{\alpha}$ are continuous at $t=t_{0}$ with respect to the Hausdorff metric $d_{H}$ for all $\alpha \in[0,1]$

Definition 2.4. A mapping $F: T \rightarrow I F_{1}$ is said to be differentiable at $t_{0}$ if there exist $F^{\prime}\left(t_{0}\right) \in I F_{1}$ such that limits:

$$
\lim _{\Delta t \rightarrow 0^{+}} \frac{F\left(t_{0}+\Delta t\right) \ominus F\left(t_{0}\right)}{\Delta t} \quad \text { and } \lim _{\Delta t \rightarrow 0^{+}} \frac{F\left(t_{0}\right) \ominus F\left(t_{0}-\Delta t\right)}{\Delta t}
$$

exist and they are equal to $F^{\prime}\left(t_{0}\right)=\left\langle u^{\prime}\left(t_{0}\right), v^{\prime}\left(t_{0}\right)\right\rangle$.

Here the limit is taken in the metric space $\left(\mathrm{IF}_{1}, d_{\infty}\right)$. At the end points of $T$ we consider only the one-sided derivatives.

If $F: T \rightarrow \mathrm{IF}_{1}$ is differentiable at $t_{0} \in T$, then we say that $F^{\prime}\left(t_{0}\right)$ is the intuitionistic fuzzy derivative of $F(t)$ at the point $t_{0}$.

Theorem 2.2 ([4]). Let $F: T \rightarrow I F_{1}$ be levelwise continuous, Then for every $t \in T$ the integral $G(t)=\int_{a}^{t} F(s) d s$ is differentiable and $G^{\prime}(t)=F(t)$.

Theorem 2.3 ([4]). Let $F: T \rightarrow I F_{1}$ be differentiable and assume that the derivative $F^{\prime}$ is integrable over $T$. Then, for each $s \in T$, we have

$$
F(s)=F(a) \oplus \int_{a}^{s} F^{\prime}(t) d t
$$




\section{Main result}

Assume that $f: I \times \mathrm{IF}_{1} \rightarrow \mathrm{IF}_{1}$ is levelwise continuous, where the interval $I=\left\{t:\left|t-t_{0}\right| \leq a\right\}$. Consider the intuitionistic fuzzy differential equation

$$
\left\{\begin{array}{l}
x^{\prime}(t)=f(t, x(t)) \\
x\left(t_{0}\right)=x_{0}
\end{array}\right.
$$

where $x_{0} \in \mathrm{IF}_{1}$.

We denote $J_{0}=I \times B\left(x_{0}, b\right)$ where $a>0, b>0, x_{0} \in \mathrm{IF}_{1}$

$$
B\left(x_{0}, b\right)=\left\{x \in \mathrm{IF}_{1} \mid d_{\infty}\left(x, x_{0}\right) \leq b\right\}
$$

with the parametric representation of $x(t), f(t, x(t))$ and $x_{0}$ are respectively

$$
\begin{gathered}
\left(\left(\underline{x}^{+}(\alpha, t), \bar{x}^{+}(\alpha, t)\right),\left(\underline{x}^{-}(\alpha, t), \bar{x}^{-}(\alpha, t)\right)\right) \\
\left(\left(\underline{f}^{+}(\alpha, t, x(\alpha, t)), \bar{f}^{+}(\alpha, t, x(\alpha, t)),\left(\underline{f}^{-}(\alpha, t, x(\alpha, t)), \bar{f}^{-}(\alpha, t, x(\alpha, t))\right)\right.\right.
\end{gathered}
$$

and

$$
\left(\left(\underline{x}^{+}(\alpha, 0), \bar{x}^{+}(\alpha, 0)\right),\left(\underline{x}^{-}(\alpha, 0), \bar{x}^{-}(\alpha, 0)\right)\right) .
$$

Definition 3.1. A mapping $x: I \rightarrow I F_{1}$ is a solution to the problem (3.1) if it is levelwise continuous and satisfies the integral equation

$$
x(t)=x_{0} \oplus \int_{t_{0}}^{t} f(s, x(s)) d s . \text { for all } t \in I
$$

i.e.,

$$
\begin{aligned}
& \underline{x}^{+}(\alpha, t)=\underline{x}^{+}\left(\alpha, t_{0}\right)+\int_{t_{0}}^{t} \underline{f}^{+}(\alpha, s, x(\alpha, s)) d s . \text { for all } t \in I \\
& \bar{x}^{+}(\alpha, t)=\bar{x}^{+}\left(\alpha, t_{0}\right)+\int_{t_{0}}^{t} \bar{f}^{+}(\alpha, s, x(\alpha, s)) d s . \text { for all } t \in I \\
& \underline{x}^{-}(\alpha, t)=\underline{x}^{-}\left(\alpha, t_{0}\right)+\int_{t_{0}}^{t} \underline{f}^{-}(\alpha, s, x(\alpha, s)) d s . \text { for all } t \in I \\
& \bar{x}^{-}(\alpha, t)=\bar{x}^{-}\left(\alpha, t_{0}\right)+\int_{t_{0}}^{t} \bar{f}^{-}(\alpha, s, x(\alpha, s)) d s . \text { for all } t \in I
\end{aligned}
$$

\section{Remark 3.1.}

- If we have a mapping $f: I \rightarrow I F_{1}$ is continuous when $I F_{1}$ is endowed with the topology generated by the metric $d_{\infty}$ implies that lower and upper $\alpha$-cuts of $f$ are continuous with respect to the Hausdorff metric $d_{H}$ which means $f$ is levelwise continuous. In fact, Let $\epsilon>0$ and $t_{0} \in I$, by continuity of $f$ there exists $a \delta>0$ such that

$$
d_{\infty}\left(F(t), F\left(t_{0}\right)\right)<\epsilon \text { whenever }\left|t-t_{0}\right|<\delta
$$

$d_{\infty}\left(F(t), F\left(t_{0}\right)\right)<\epsilon \Rightarrow$ 


$$
\left|[(F(t))]_{r}^{+}(\alpha)-\left[F\left(t_{0}\right)\right]_{r}^{+}(\alpha)\right|<\epsilon \text { and }\left|[F(t)]_{l}^{+}(\alpha)-\left[F\left(t_{0}\right)\right]_{l}^{+}(\alpha)\right|<\epsilon
$$

hence

$\max \left(\left|[(F(t))]_{r}^{+}(\alpha)-\left[F\left(t_{0}\right)\right]_{r}^{+}(\alpha)\right| ;\left|[F(t)]_{l}^{+}(\alpha)-\left[F\left(t_{0}\right)\right]_{l}^{+}(\alpha)\right|\right)=d_{H}\left(F_{\alpha}(t), F_{\alpha}\left(t_{0}\right)\right)<\epsilon$ whenever $\left|t-t_{0}\right|<\delta$. So $F_{\alpha}(t)$ is continuous with respect to the Hausdorff metric. The same for $F^{\alpha}(t)$.

- The Theorem 2.2 and Theorem 2.3 prove the equivalence between Cauchy problem (3.1) and integral equation (3.2)

Theorem 3.1 ([3]). Assume that $f$ is levelwise continuous and there exists a constant $k>0$ such that

$$
\begin{aligned}
\left|[f(s, x(s))]_{r}^{+}(\alpha)-[f(s, y(s))]_{r}^{+}(\alpha)\right| & \leq k\left|[x(s)]_{r}^{+}(\alpha)-[y(s)]_{r}^{+}(\alpha)\right| \\
\left|[f(s, x(s))]_{l}^{+}(\alpha)-[f(s, y(s))]_{l}^{+}(\alpha)\right| & \leq k\left|[x(s)]_{l}^{+}(\alpha)-[y(s)]_{l}^{+}(\alpha)\right| \\
\left|[f(s, x(s))]_{r}^{-}(\alpha)-[f(s, y(s))]_{r}^{-}(\alpha)\right| & \leq k\left|[x(s)]_{r}^{-}(\alpha)-[y(s)]_{r}^{-}(\alpha)\right| \\
\left|[f(s, x(s))]_{l}^{-}(\alpha)-[f(s, y(s))]_{l}^{-}(\alpha)\right| & \leq k\left|[x(s)]_{l}^{-}(\alpha)-[y(s)]_{l}^{-}(\alpha)\right|
\end{aligned}
$$

with $k\left(T-t_{0}\right)<1$, for all $s \in I, x, y \in I F_{1}$. Then the initial value problem (3.1) has a unique solution on $I$.

\subsection{Picard's approximation method}

Let $f$ be levelwise continuous in a region $J_{0}$ containing the point $\left(t_{0}, x_{0}\right)$ By integrating both sides of the differential equation (3.1) with respect to $t$, we get

$$
\begin{aligned}
& \underline{x}^{+}(\alpha, t)=\underline{c}^{+}+\int_{t_{0}}^{t} \underline{f}^{+}(\alpha, s, x(\alpha, s)) d s . \text { for all } t \in I \\
& \bar{x}^{+}(\alpha, t)=\bar{c}^{+}+\int_{t_{0}}^{t} \bar{f}^{+}(\alpha, s, x(\alpha, s)) d s . \text { for all } t \in I \\
& \underline{x}^{-}(\alpha, t)=\underline{c}^{-}+\int_{t_{0}}^{t} \underline{f}^{-}(\alpha, s, x(\alpha, s)) d s . \text { for all } t \in I \\
& \bar{x}^{-}(\alpha, t)=\bar{c}^{-}+\int_{t_{0}}^{t} \bar{f}^{-}(\alpha, s, x(\alpha, s)) d s . \text { for all } t \in I
\end{aligned}
$$

where $\underline{c}^{+}, \bar{c}^{+}, \underline{c}^{-}$and $\bar{c}^{-}$are constants.

We replace $t$ by $t_{0}$, we obtain that $\underline{c}^{+}=\underline{x}^{+}\left(\alpha, t_{0}\right), \bar{c}^{+}=\bar{x}^{+}\left(\alpha, t_{0}\right), \underline{c}^{-}=\underline{x}^{-}\left(\alpha, t_{0}\right)$ and $\bar{c}^{-}=\bar{x}^{-}\left(\alpha, t_{0}\right)$ Thus,

$$
\begin{aligned}
& \underline{x}^{+}(\alpha, t)=\underline{x}^{+}\left(\alpha, t_{0}\right)+\int_{t_{0}}^{t} \underline{f}^{+}(\alpha, s, x(\alpha, s)) d s . \\
& \bar{x}^{+}(\alpha, t)=\bar{x}^{+}\left(\alpha, t_{0}\right)+\int_{t_{0}}^{t} \bar{f}^{+}(\alpha, s, x(\alpha, s)) d s .
\end{aligned}
$$




$$
\begin{gathered}
\underline{x}^{-}(\alpha, t)=\underline{x}^{-}\left(\alpha, t_{0}\right)+\int_{t_{0}}^{t} \underline{f}^{-}(\alpha, s, x(\alpha, s)) d s \\
\bar{x}^{-}(\alpha, t)=\bar{x}^{-}\left(\alpha, t_{0}\right)+\int_{t_{0}}^{t} \bar{f}^{-}(\alpha, s, x(\alpha, s)) d s \text { for all } t \in I
\end{gathered}
$$

Now to get power series solution of (3.2) we will use Picard's successive approximation method.

Suppose $x_{0}(t)$ be an arbitrary levelwise continuous function that represents an approximation to the solution of (3.2). Since $f\left(t, x_{0}(t)\right)$ is a known function depending solely on $x$ it can be integrated with $x(t)$ replaced by $x_{0}(t)$. The right hand side of (3.2) defines another function, which we write as

$$
\begin{aligned}
& \underline{x}_{1}^{+}(\alpha, t)=\underline{x}^{+}\left(\alpha, t_{0}\right)+\int_{t_{0}}^{t} \underline{f}^{+}\left(\alpha, s, x_{0}(\alpha, s)\right) d s . \\
& \bar{x}_{1}^{+}(\alpha, t)=\bar{x}^{+}\left(\alpha, t_{0}\right)+\int_{t_{0}}^{t} \bar{f}^{+}\left(\alpha, s, x_{0}(\alpha, s)\right) d s . \\
& \underline{x}_{1}^{-}(\alpha, t)=\underline{x}^{-}\left(\alpha, t_{0}\right)+\int_{t_{0}}^{t} \underline{f}^{-}\left(\alpha, s, x_{0}(\alpha, s)\right) d s . \\
& \bar{x}_{1}^{-}(\alpha, t)=\bar{x}^{-}\left(\alpha, t_{0}\right)+\int_{t_{0}}^{t} \bar{f}^{-}\left(\alpha, s, x_{0}(\alpha, s)\right) d s .
\end{aligned}
$$

for all $t \in I$.

When we repeat the procedure, we obtain a sequence of functions $x_{1}(t), x_{2}(t), x_{3}(t), \ldots$, whose $n$-th term is defined by the relation

$$
\begin{aligned}
& \underline{x}_{n}^{+}(\alpha, t)=\underline{x}^{+}\left(\alpha, t_{0}\right)+\int_{t_{0}}^{t} \underline{f}^{+}\left(\alpha, s, x_{n-1}(\alpha, s)\right) d s . \\
& \bar{x}_{n}^{+}(\alpha, t)=\bar{x}^{+}\left(\alpha, t_{0}\right)+\int_{t_{0}}^{t} \bar{f}^{+}\left(\alpha, s, x_{n-1}(\alpha, s)\right) d s . \\
& \underline{x}_{n}^{-}(\alpha, t)=\underline{x}^{-}\left(\alpha, t_{0}\right)+\int_{t_{0}}^{t} \underline{f}^{-}\left(\alpha, s, x_{n-1}(\alpha, s)\right) d s . \\
& \bar{x}_{n}^{-}(\alpha, t)=\bar{x}^{-}\left(\alpha, t_{0}\right)+\int_{t_{0}}^{t} \bar{f}^{-}\left(\alpha, s, x_{n-1}(\alpha, s)\right) d s .
\end{aligned}
$$

for all $t \in I n=1,2,3, \ldots$

Theorem 3.2. Let the exact solution $\left\{\left(\underline{X}^{+}(\alpha, t), \overline{X^{+}}(\alpha, t)\right),\left(\underline{X}^{-}(\alpha, t), \overline{X^{-}}(\alpha, t)\right)\right\}$ be approximated by $\left\{\left(\underline{x}^{+}(\alpha, t), \overline{x^{+}}(\alpha, t)\right),\left(\underline{x}^{-}(\alpha, t), \overline{x^{-}}(\alpha, t)\right)\right\}$ For arbitrarily fixed $0<\alpha \leq 1$, the solution of (3.1) using Picard's approximation method 3.1 converges to the exact solutions $\underline{X}^{+}(\alpha, t)$, $\overline{X^{+}}(\alpha, t) \underline{X}^{-}(\alpha, t), \overline{X^{-}}(\alpha, t)$ uniformly in $t$.

Proof. It is sufficient to show that

$$
\underline{X}^{+}(\alpha, t)=\lim _{n \rightarrow \infty} \underline{x}_{n}^{+}(\alpha, t)
$$




$$
\begin{aligned}
& \bar{X}^{+}(\alpha, t)=\lim _{n \rightarrow \infty} \bar{x}_{n}^{+}(\alpha, t) \\
& \underline{X}^{-}(\alpha, t)=\lim _{n \rightarrow \infty} \underline{x}_{n}^{-}(\alpha, t)
\end{aligned}
$$

and

$$
\bar{X}^{-}(\alpha, t)=\lim _{n \rightarrow \infty} \bar{x}_{n}^{-}(\alpha, t)
$$

for all $0 \leq \alpha \leq 1$

Let the exact solution of (3.1) be

$$
X(\alpha, t)=X(\alpha, 0)+\int_{0}^{t} f(\alpha, s, X(s)) d s
$$

and the solution of (3.1) using Picard's approximation method is

$$
x_{n}(\alpha, t)=x(\alpha, 0)+\int_{0}^{t} f\left(\alpha, s, x_{n-1}(s)\right) d s
$$

Now, we denote

$$
\begin{aligned}
& l_{n}^{+}=\underline{X}^{+}(\alpha, t)-\underline{x}_{n}^{+}(\alpha, t) \\
& u_{n}^{+}=\bar{X}^{+}(\alpha, t)-\bar{x}_{n}^{+}(\alpha, t) \\
& l_{n}^{-}=\underline{X}^{-}(\alpha, t)-\underline{x}_{n}^{-}(\alpha, t) \\
& u_{n}^{-}=\bar{X}^{-}(\alpha, t)-\bar{x}_{n}^{-}(\alpha, t)
\end{aligned}
$$

Using (3.3) and (3.4), we have

$$
\begin{aligned}
l_{n}^{+} & =\underline{X}^{+}(\alpha, 0)+\int_{0}^{t} \underline{f}^{+}\left(\alpha, s, \underline{X}^{+}(s)\right) d s-\underline{x}^{+}(\alpha, 0)-\int_{0}^{t} \underline{f}^{+}\left(\alpha, s, \underline{x}_{n-1}^{+}(s)\right) d s \\
& =\underline{X}^{+}(\alpha, 0)-\underline{x}^{+}(\alpha, 0)+\int_{0}^{t}\left(\underline{f}^{+}\left(\alpha, s, \underline{X}^{+}(s)\right)-\underline{f}^{+}\left(\alpha, s, \underline{x}_{n-1}^{+}(s)\right)\right) d s \\
& \left.\left.\leq l_{0}^{+}+k \int_{0}^{t}\left(\underline{X}^{+}(\alpha, s)\right)-\underline{x}_{n-1}^{+}(\alpha, s)\right)\right) d s
\end{aligned}
$$

Since $\left.l_{0}^{+}=\underline{X}^{+}(\alpha, 0)-\underline{x}^{+}(\alpha, 0)=0, \underline{X}^{+}(\alpha, s)\right)-\underline{x}_{0}^{+}(\alpha, s) \leq M t$

where $M=\underline{f}^{+}\left(\alpha, s, \underline{X}^{+}(s)\right)-\underline{0}_{\langle 1,0\rangle}^{+}(\alpha, s)$ We have $l_{1}^{+} \leq K M t$ and $l_{2}^{+} \leq \frac{M K^{2} t^{2}}{2}$

So, we can assume $l_{n}^{+} \leq \frac{M K^{n} t^{n}}{n !}$ and this can be easily proved by induction.

Therefore, $\lim _{n \rightarrow \infty} l_{n}^{+} \leq \lim _{n \rightarrow \infty} \frac{M K^{n} t^{n}}{n !}=0$ i.e $\lim _{n \rightarrow \infty} l_{n}^{+}=\lim _{n \rightarrow \infty} \underline{X}^{+}(\alpha, t)-\underline{x}_{n}^{+}(\alpha, t)=0$

Thus, $\lim _{n \rightarrow \infty} \underline{x}_{n}^{+}(\alpha, t)=\underline{X}^{+}(\alpha, t)$

Similarly we can prove $\lim _{n \rightarrow \infty} \bar{x}_{n}^{+}(\alpha, t)=\bar{X}^{+}(\alpha, t), \lim _{n \rightarrow \infty} \underline{x}_{n}^{-}(\alpha, t)=\underline{X}^{-}(\alpha, t)$

and $\lim _{n \rightarrow \infty} \bar{x}_{n}^{-}(\alpha, t)=\bar{X}^{-}(\alpha, t)$. 


\section{Numerical example}

Consider the intuitionistic fuzzy initial value problem

$$
\left\{\begin{array}{l}
x^{\prime}(t)=x(t), t \in I=[0,1] \\
x(0)=((0.75+0.25 \alpha, 1.125-0.125 \alpha) ;(0.5+0.5 \alpha, 2-\alpha))
\end{array}\right.
$$

To solve this IFIVP first we transform the problem into crisp system of ordinary initial value problems

$$
\begin{cases}{\underline{x^{\prime+}}}^{+}(\alpha, t)=\underline{x}^{+}(\alpha, t) & ; \underline{x}^{+}(\alpha, 0)=0.75+0.25 \alpha \\ \bar{x}^{+}(\alpha, t)=\bar{x}^{+}(\alpha, t), & ; \bar{x}^{+}(\alpha, 0)=0.25 \alpha \\ {\underline{x^{\prime}}}^{-}(\alpha, t)=\underline{x}^{-}(\alpha, t), & ; \underline{x}^{-}(\alpha, 0)=0.5+0.5 \alpha \\ \overline{x^{\prime}}(\alpha, t)=\bar{x}^{-}(\alpha, t), & ; \bar{x}^{-}(\alpha, 0)=2-\alpha\end{cases}
$$

the parametric form of the exact solution is

$$
\left\{\begin{array}{l}
\underline{X}^{+}(\alpha, t)=\underline{x}^{+}(\alpha, 0) \exp (t) \\
\bar{X}^{+}(\alpha, t)=\bar{x}^{+}(\alpha, 0) \exp (t) \\
\underline{X}^{-}(\alpha, t)=\underline{x}^{-}(\alpha, 0) \exp (t) \\
\bar{X}^{-}(\alpha, t)=\bar{x}^{-}(\alpha, 0) \exp (t)
\end{array}\right.
$$

for all $0<\alpha \leq 1$.

The approximate solution using Picard's method for $n$-times is

$$
\left\{\begin{array}{l}
\underline{x}^{+}(\alpha, t)=\underline{x}^{+}(\alpha, 0)\left(1+t+\frac{t^{2}}{2}+\frac{t^{3}}{3 !}+\ldots\right), \\
\bar{x}^{+}(\alpha, t)=\bar{x}^{+}(\alpha, 0)\left(1+t+\frac{t^{2}}{2}+\frac{t^{3}}{3 !}+\ldots\right), \\
\underline{x}^{-}(\alpha, t)=\underline{x}^{-}(\alpha, 0)\left(1+t+\frac{t^{2}}{2}+\frac{t^{3}}{3 !}+\ldots\right), \\
\bar{x}^{-}(\alpha, t)=\bar{x}^{-}(\alpha, 0)\left(1+t+\frac{t^{2}}{2}+\frac{t^{3}}{3 !}+\ldots\right)
\end{array}\right.
$$

for all $0<\alpha \leq 1$.

The exact and approximate solutions of IFIVP (4.1) for $n=2, n=3$ and $n=4$ are compared and plotted at $t=1$ shown in Figs 1, 2 and 3.

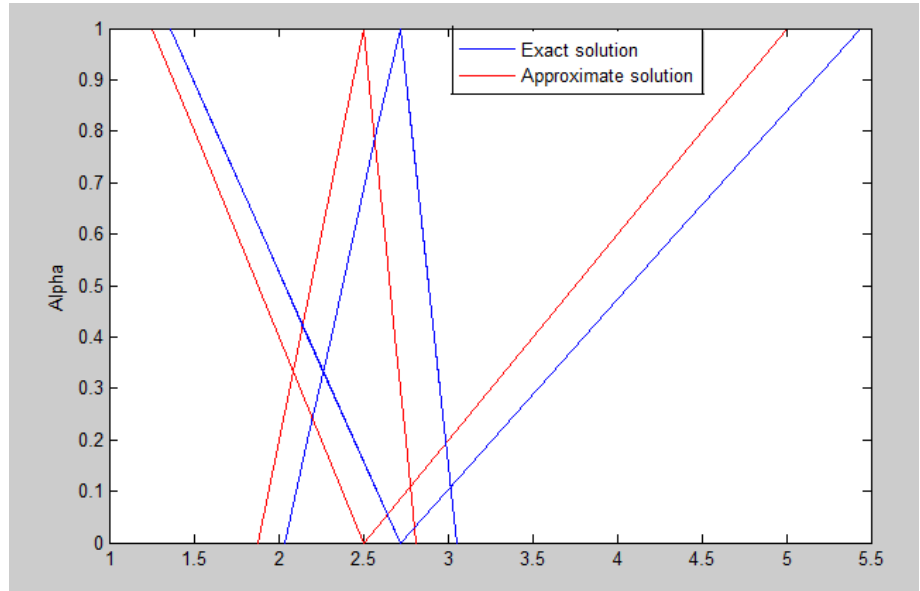

Figure 1. $n=2$ 


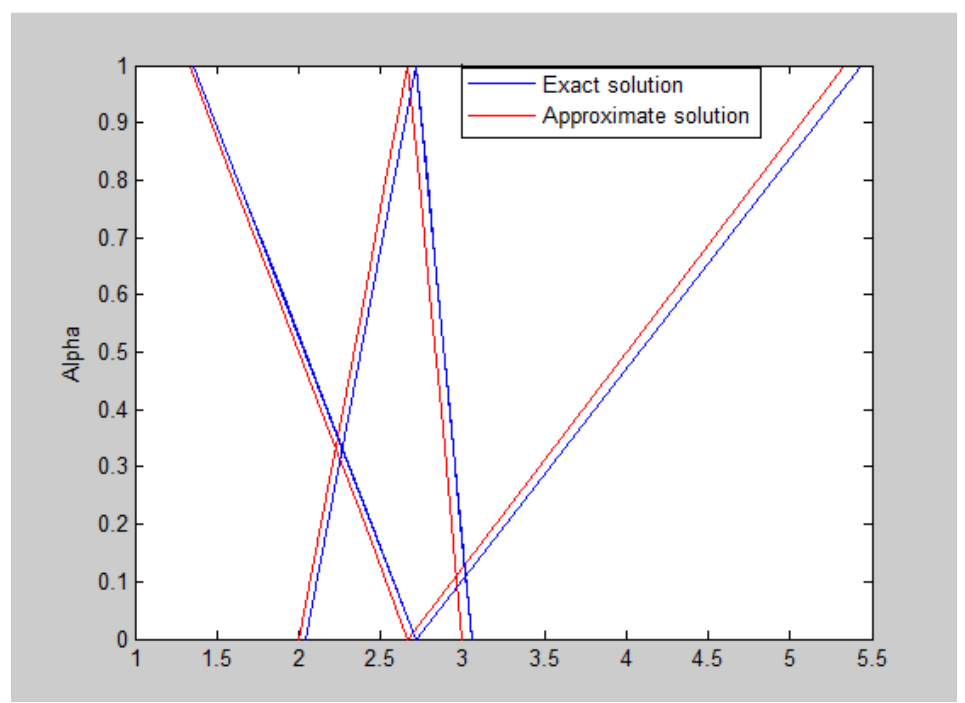

Figure 2. $n=3$

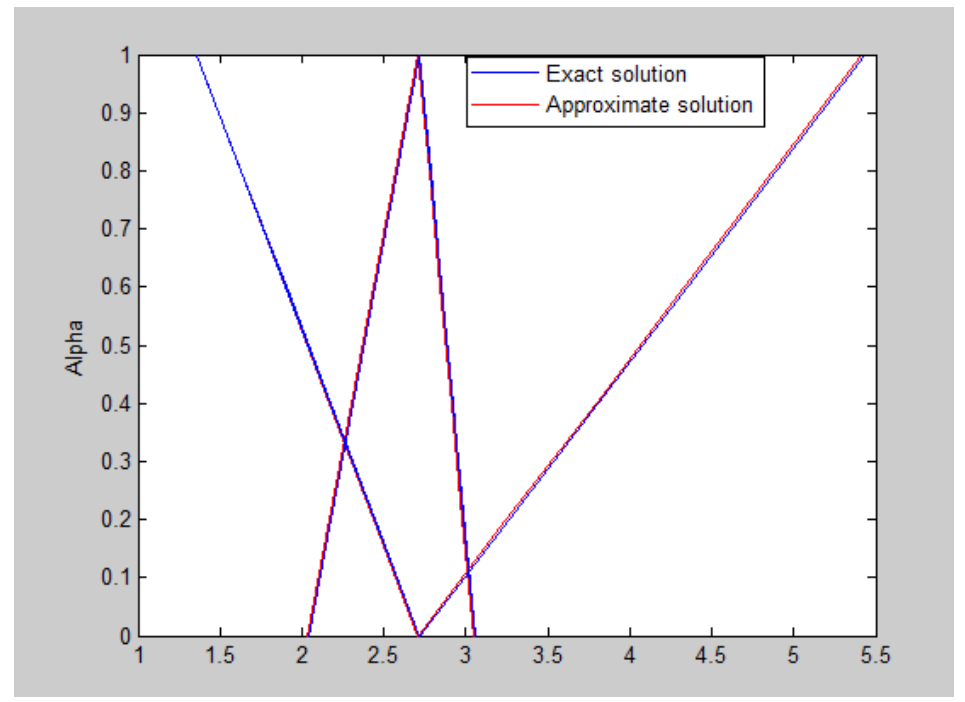

Figure 3. $n=4$

\section{References}

[1] Atanassov, K. (1986) Intuitionistic fuzzy sets, Fuzzy Sets and Systems, 20, 87-96.

[2] Atanassov, K. (1999) Intuitionistic Fuzzy Sets: Theory and Applications, Springer PhysicaVerlag, Heidelberg.

[3] Ettoussi, R., Melliani, S., \& Chadli, L. S. (2017) Differential equation with intuitionistic fuzzy parameters, Notes on Intuitionistic Fuzzy Sets, 23(4), 46-61.

[4] Ettoussi, R., Melliani, S., Elomari, M., \& Chadli, L. S. (2015) Solution of intuitionistic fuzzy differential equations by successive approximations method, Notes on Intuitionistic Fuzzy Sets, 21(2), 51-62.

[5] Keyanpour, M., \& Akbarian, T. (2014) Solving Intuitionistic Fuzzy Nonlinear Equations, Journal of Fuzzy Set Valued Analysis, 2014, 1-6. 
[6] Melliani, S., \& Chadli, L. S. (2001) Introduction to intuitionistic fuzzy partial differential Equations, Notes on Intuitionistic Fuzzy Sets, 7(3), 39-42.

[7] Melliani, S., Elomari, M., Chadli, L. S., \& Ettoussi, R. (2015) Intuitionistic Fuzzy metric spaces, Notes on intuitionistic Fuzzy Sets, 21(1), 43-53.

[8] Zadeh, L. A. (1965) Fuzzy set, Information and Control, 8(3), 338-353. 